\title{
A Geometric Proof of the Colored Tverberg Theorem
}

\author{
Jiří Matoušek · Martin Tancer • Uli Wagner
}

Received: 30 August 2010 / Revised: 7 June 2011 / Accepted: 7 June 2011 /

Published online: 12 July 2011

(C) Springer Science+Business Media, LLC 2011

\begin{abstract}
The colored Tverberg theorem asserts that for every $d$ and $r$ there exists $t=t(d, r)$ such that for every set $C \subset \mathbb{R}^{d}$ of cardinality $(d+1) t$, partitioned into $t$ point subsets $C_{1}, C_{2}, \ldots, C_{d+1}$ (which we think of as color classes; e.g., the points of $C_{1}$ are red, the points of $C_{2}$ blue, etc.), there exist $r$ disjoint sets $R_{1}, R_{2}, \ldots, R_{r} \subseteq C$ that are rainbow, meaning that $\left|R_{i} \cap C_{j}\right| \leq 1$ for every $i, j$, and whose convex hulls all have a common point.

All known proofs of this theorem are topological. We present a geometric version of a recent beautiful proof by Blagojević, Matschke, and Ziegler, avoiding a direct use of topological methods. The purpose of this de-topologization is to make the proof more concrete and intuitive, and accessible to a wider audience.
\end{abstract}

Keywords Convexity · Tverberg's theorem · Colored Tverberg theorem · Continuous motion

\section{Introduction}

We first recall three fundamental results of discrete geometry, all of them dealing with partitioning finite sets in $\mathbb{R}^{d}$ so that the convex hulls of the parts intersect. In the order of increasing sophistication, they are Radon's lemma, Tverberg's theorem, and

J. Matoušek · M. Tancer

Department of Applied Mathematics, Charles University, Malostranské nám. 25, 11800 Praha 1,

Czech Republic

J. Matoušek $\cdot$ M. Tancer

Institute of Theoretical Computer Science (ITI), Charles University, Malostranské nám. 25,

11800 Praha 1, Czech Republic

J. Matoušek · U. Wagner $(\bowtie)$

Institute of Theoretical Computer Science, ETH Zürich, 8092 Zürich, Switzerland

e-mail: wagneru@inf.ethz.ch 


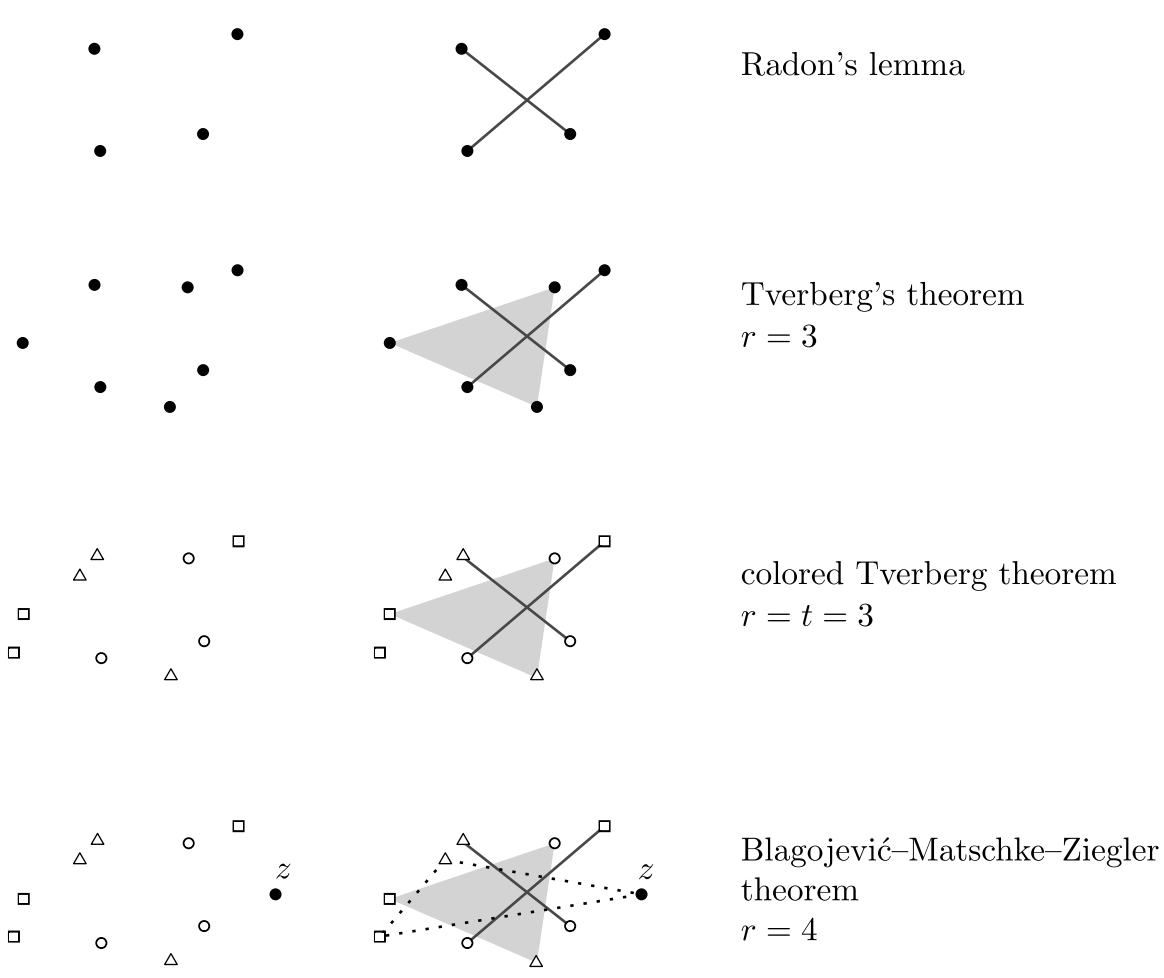

Fig. 1 Radon's lemma, Tverberg's theorem, the colored Tverberg theorem, and the BlagojevićMatschke-Ziegler theorem: planar illustrations

the colored Tverberg theorem. We refer to [11] for more background, applications, and historical references not mentioned here.

Radon's Theorem asserts that every set $C \subset \mathbb{R}^{d}$ of $d+2$ points has two disjoint subsets $A_{1}, A_{2}$ with $\operatorname{conv}\left(A_{1}\right) \cap \operatorname{conv}\left(A_{2}\right) \neq \emptyset$; see the illustration of the planar case in Fig. 1. The proof is simple linear algebra.

Tverberg's Theorem states that every set $C \subset \mathbb{R}^{d}$ of $(d+1)(r-1)+1$ points has $r$ pairwise disjoint subsets $A_{1}, \ldots, A_{r}$ with $\bigcap_{i=1}^{r} \operatorname{conv}\left(A_{i}\right) \neq \varnothing$ (so Radon's lemma is the $r=2$ case). Several geometric proofs are known, e.g., [14-16]. The number $(d+1)(r-1)+1$ is easily shown to be the smallest possible for such a claim to hold, e.g., by considering the configuration $C$ of $(d+1)(r-1)$ points forming $d+1$ small clusters by $r-1$ points each, as in Fig. 2 .

It is easy to show (e.g., by iterating Radon's lemma) that there exists some number $T=T(d, r)$ such that the conclusion of the theorem holds for every set $C$ with at least $T$ points. The hard part of Tverberg's theorem is obtaining the optimal value of $T(d, r)$.

The Colored Tverberg Theorem has a setting similar to that of Tverberg's theorem. Again we have a set $C \subset \mathbb{R}^{d}$ and seek $r$ pairwise disjoint subsets whose convex hulls all share a point, but this time these subsets have to satisfy an additional restriction. 
Fig. 2 A configuration with no Tverberg $r$-partition

$$
\because r-1
$$
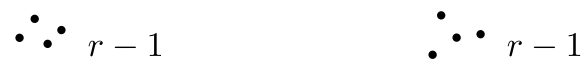

We introduce the following terminology. Let $C \subset \mathbb{R}^{d}$ be a finite set partitioned into $k$ color classes $C_{1}, C_{2}, \ldots, C_{k}$ (in other words, each point of $C$ is colored by one of $k$ colors). A subset $R \subseteq C$ is rainbow if it contains at most one point of each color, i.e., $\left|R \cap C_{j}\right| \leq 1$ for all $j$.

A rainbow $r$-partition for $C$ is an ordered $r$-tuple $\mathcal{R}=\left(R_{1}, \ldots, R_{r}\right)$ of pairwise disjoint rainbow subsets of $C$. We stress that, for technical convenience, and with a mild abuse of the terminology "partition", we generally do not require that the $R_{i}$ cover all of $C$ (if they do, we speak of a maximal rainbow r-partition).

A rainbow $r$-partition is Tverberg if it has a Tverberg point, i.e., a point $x \in$ $\bigcap_{i=1}^{r} \operatorname{conv}\left(R_{i}\right)$ (which usually does not belong to $C$ ). The colored Tverberg theorem can then be stated as follows.

Theorem 1 (Colored Tverberg theorem) For every $d \geq 1$ and $r \geq 2$ there exists $t$ such that whenever $C \subset \mathbb{R}^{d}$ is a set of $(d+1) t$ points partitioned into $t$-point subsets $C_{1}, \ldots, C_{d+1}$, then there is a Tverberg rainbow $r$-partition $\mathcal{R}=\left(R_{1}, \ldots, R_{r}\right)$ for $C$.

The theorem is usually stated with $\mathcal{R}$ maximal, in which case each $R_{i}$ has to be a $(d+1)$-element set containing one point of each color. However, we chose to omit maximality, since on the one hand, the proof typically does not yield a maximal $\mathcal{R}$, and on the other hand, some thought reveals that, in the situation of Theorem 1, an arbitrary $\mathcal{R}$ can easily be extended into a maximal one.

For the colored Tverberg theorem, proving the existence of any $t$, no matter how large, seems difficult, and the simplest proof currently known is also the one that yields the smallest $t$, as we will briefly discuss below.

Let $t(d, r)$ denote the smallest $t$ for which the conclusion of the theorem holds. The configuration with $d+1$ clusters by $r-1$ points each, as in Fig. 2, where the $i$ th cluster is all colored with color $i$, shows that $t(d, r) \geq r$.

Historical Notes The validity of the colored Tverberg theorem was first conjectured by Bárány, Füredi and Lovász [4], who proved the case $d=2, r=3$, obtaining $t(2,3) \leq 7$. Bárány and Larman [2] settled the planar case, showing $t(2, r)=r$ for all $r$ (their paper also contains Lovász' topological proof showing that $t(d, 2)=2$ for all $d$ ). They conjectured that $t(d, r)=r$ for all $r, d$.

The first proof of the general case of the colored Tverberg theorem was obtained by Živaljević and Vrećica [21] (simpler versions were provided in [5, 10]). Their proof is topological, and it builds on the pioneering works by Bajmóczy and Bárány [1] (who gave a new, topological proof of Radon's lemma) and by Bárány, Shlosman, 
and Szúcs [3] (who provided a topological proof of Tverberg's theorem assuming that $r$ is a prime number).

The Živaljević-Vrećica method yields $t(d, r) \leq 2 r-1$ for all prime $r$. Later, the same bound was extended to all $r$ that are prime powers [20], using more advanced topological tools introduced to combinatorial geometry by Özaydin, by Volovikov, and by Sarkaria.

The most important progress by far since the 1992 Živaljević-Vrećica proof was achieved by Blagojević, Matschke, and Ziegler [6] in 2009. They discovered a new proof, also topological, which yields the optimal bound $t(d, r)=r$ whenever $r+1$ is a prime number.

Their main trick is both simple and surprising; at first sight, it seems strange that it might help in such a radical way. Namely, to the point set $C=C_{1} \cup \cdots \cup C_{d+1}$ as in the colored Tverberg theorem, with $\left|C_{1}\right|=\cdots=\left|C_{d+1}\right|=t$, they first add an (arbitrary) extra point $z$, and color it with a new color $d+2$, thus forming a singleton color class $C_{d+2}=\{z\}$. Then they prove the existence of a Tverberg rainbow $(r+1)$ partition $\left(R_{1}, \ldots, R_{r+1}\right)$ for the set $C^{\prime}=C_{1} \cup C_{2} \cup \cdots \cup C_{d+1} \cup C_{d+2}$. Given such an $(r+1)$-partition, one can simply delete the set $R_{i}$ containing the artificial point $z$, and be left with a Tverberg $r$-partition for the original $C$; see the bottom part of Fig. 1.

We now formulate the main claim of the Blagojevic et al. proof. With $r$ and $d$ fixed, let us call a $(d+2)$-tuple $\mathcal{C}=\left(C_{1}, \ldots, C_{d+2}\right)$ of pairwise disjoint sets in $\mathbb{R}^{d}$ a $B M Z$-collection (BMZ standing for Blagojević-Matschke-Ziegler) if $\left|C_{1}\right|=\left|C_{2}\right|=$ $\cdots=\left|C_{d+1}\right|=r-1$ and $\left|C_{d+2}\right|=1$.

Theorem 2 (Blagojević-Matschke-Ziegler theorem) For every $d \geq 1$ and every prime number $r$, every BMZ-collection $\mathcal{C}$ admits a Tverberg rainbow $r$-partition $\left(R_{1}, \ldots, R_{r}\right)$.

We note that for proving the colored Tverberg theorem with $r=r_{0}$, one uses the Blagojević-Matschke-Ziegler theorem with $r=r_{0}+1$.

Theorem 2 was first proved, in a preliminary version of [6], using relatively heavy topological machinery, by computing a certain obstruction in cohomology (this method also yields additional results; see [7]). Then Vrećica and Živaljević [17] found a simpler, degree-theoretic proof, and independently, the authors of [6] obtained a similar simplification.

We should remark that the Blagojević-Matschke-Ziegler theorem has a more general version, which is perhaps even nicer and more natural. Namely, for $r$ prime, whenever $N+1$ points in $\mathbb{R}^{d}, N=(d+1)(r-1)$, are partitioned into classes $C_{1}, \ldots, C_{m}, m \geq d+1$, with each $C_{i}$ of size at most $r-1$, then there is a Tverberg rainbow $r$-partition. This, for instance, also contains the original Tverberg theorem as a special case. For simplicity, though, we will consider only Theorem 2 in the rest of this paper.

This Paper Our main purpose is to present an elementary and self-contained geometric proof of Theorem 2 (and thus of the colored Tverberg theorem as well). We follow the basic strategy of the degree-theoretic proof in $[6,17]$. However, we replace 
the abstract deleted product construction by a concrete geometric construction due to Sarkaria [14] (with a simplification by Onn).

In this way, the basic scheme of the proof is clear and intuitive. A rigorous elementary presentation avoiding topological tools is not entirely simple, however, mainly because we have to deal with various issues of general position. These issues do not arise in the topological proof, since they are dealt with on a general level when building the topological apparatus.

One can say that our proof is a "de-topologized" version of the proofs in $[6,17]$. In a similar sense, Sarkaria's proof [14] of Tverberg's theorem can be regarded as a de-topologized version of his earlier topological proof of Tverberg's theorem [13]. It has become one of the most cited proofs, and often it is regarded as the standard proof, see, e.g., [9, Sect. 1.2], [8, p. 30b]. ${ }^{1}$

Another example of de-topologization is a combinatorial proof of Kneser's conjecture [12]; developing this approach further, Ziegler [18] was able to prove all known generalizations of Kneser's conjecture, plus some new ones, in a combinatorial way.

We hope that an elementary, de-topologized proof of the colored Tverberg theorem will stimulate further research by making the proof more intuitive and concrete and accessible to a wider audience. For example, this might help in attacking the open cases of the Bárány-Larman conjecture (the validity of the claim of Theorem 2 for non-prime $r$ ).

While all known topological proofs of Tverberg's theorem work only for $r$ that is a prime power, Sarkaria's de-topologized proof [14] overcomes this restriction and works for all $r$. Unfortunately, our de-topologization does not help in removing the restriction of prime $r$ in Theorem 2. If anything, it helps in seeing more clearly why the proof method of Blagojević et al. fails whenever $r$ is not a prime; see Sect. 8 for a discussion.

\section{Outline of the Proof}

Here we sketch the main steps of the proof, proceeding informally and glossing over many details.

We begin with a fixed BMZ-collection $\mathcal{C}=\left(C_{1}, \ldots, C_{d+1}, C_{d+2}=\{z\}\right)$. We assume that the points of $\mathcal{C}$ are in a sufficiently general position; if they are not, we use a standard perturbation argument.

We consider the system $\mathbf{R}=\mathbf{R}(\mathcal{C})$ of all the maximal rainbow $r$-partitions $\mathcal{R}=$ $\left(R_{1}, \ldots, R_{r}\right)$ for $\mathcal{C}$, Tverberg or not, for which $z \in R_{r}$.

Using a construction as in Sarkaria [14], with each $\mathcal{R} \in \mathbf{R}$ we associate an $N$ dimensional simplex $S_{\mathcal{R}}$ in $\mathbb{R}^{N}$. (More precisely, some of the $S_{\mathcal{R}}$ may be degenerate, i.e., only $(N-1)$-dimensional, even for $\mathcal{C}$ in general position, but this will not matter-so for the purposes of this outline, we pretend that they are all $N$ dimensional.) The key property of this construction is that $\mathcal{R}$ is Tverberg iff $S_{\mathcal{R}}$ contains the origin 0 .

\footnotetext{
${ }^{1}$ We remark that another possible strategy for de-topologizing Tverberg-type statements was suggested in [19]. The so-called guiding principle on p. 94 of that paper suggests a certain way of relaxing the symmetry (equivariance) condition to obtain a more general geometric statement that might be more amenable to a purely geometric proof in the spirit of Sarkaria's proof of the geometric Tverberg theorem.
} 
Fig. 3 A schematic illustration of the situation in $\mathbb{R}^{N}$

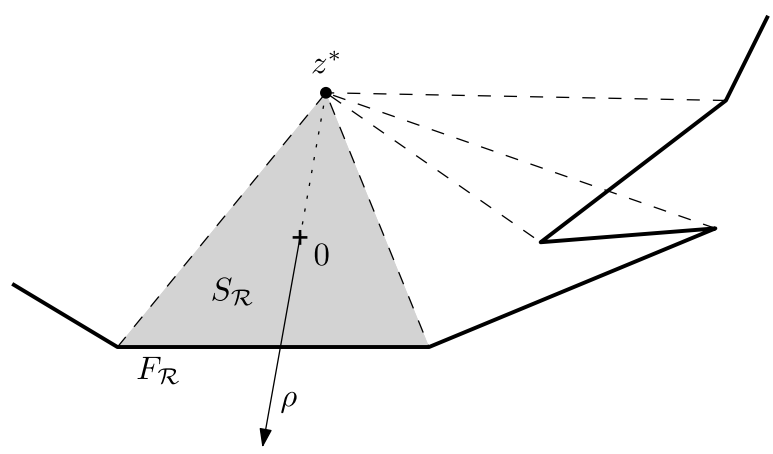

Fig. 4 Defining the degree of $\Sigma$; the positive side of $\Sigma$ is marked gray

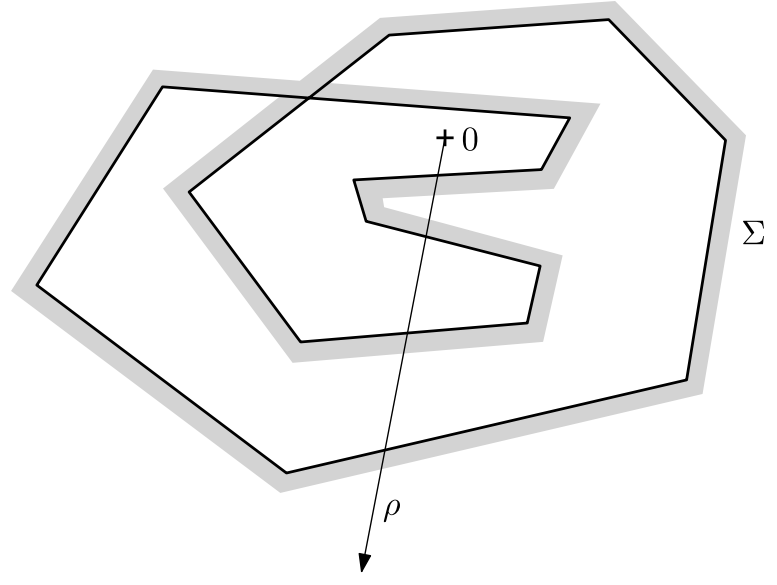

Moreover, all of the $S_{\mathcal{R}}$ have one vertex $z^{*}$ in common. Let $F_{\mathcal{R}}$ be the facet of $S_{\mathcal{R}}$ opposite to $z^{*}$; this is an $(N-1)$-dimensional simplex avoiding 0 . Then we find that $\mathcal{R}$ is Tverberg iff the ray $\rho$ emanating from 0 in the direction opposite to $0 z^{*}$ meets $F_{\mathcal{R}}$; see Fig. 3 for a schematic planar illustration.

Next, it turns out that the union $\Sigma$ of all the $F_{\mathcal{R}}$ forms something like a (possibly self-intersecting) hypersurface in $\mathbb{R}^{N}$, and one can define the degree of $\Sigma$, a standard notion in topology. (Since $\Sigma$ is determined by $\mathcal{C}$, we also speak of the degree of $\mathcal{C}$ and write $\operatorname{deg}(\mathcal{C})$.)

Intuitively, the degree counts how many times $\Sigma$ "winds" around 0 . Its absolute value is a lower bound for the number of times a ray like $\rho$ intersects $\Sigma$. Thus, if we can show that the degree of $\Sigma$ is always nonzero, then $\rho$ has to intersect at least one $F_{\mathcal{R}}$, and the existence of a Tverberg rainbow $r$-partition follows.

First we need to equip $\Sigma$ with an orientation, which means designating one of the "sides" of $\Sigma$ as positive and the other as negative; see Fig. 4. The orientation is defined locally: we determine positive and negative side for every $F_{\mathcal{R}}$, in a globally 
consistent way. The definitions must match at the "seams" where two of the $F_{\mathcal{R}}$ 's meet in an $(N-2)$-dimensional face. ${ }^{2}$

Then we define the degree of $\Sigma$ as the number of times the ray $\rho$ passes from the negative side of $\Sigma$ to the positive side minus the number of times it passes from the positive side to the negative one (in the picture, the degree is +2 ). As expected, the degree does not depend on the choice of the ray $\rho$-any other ray emanating from 0 yields the same number.

It remains to verify that $\operatorname{deg}(\mathcal{C}) \neq 0$, and this is done by a "continuous motion" argument. Namely, we fix a special BMZ-collection $\mathcal{C}_{0}$ for which the degree can be explicitly computed. Then we consider a continuous motion of the points of $\mathcal{C}_{0}$ that transforms it to the given BMZ-collection $\mathcal{C}$. We follow the corresponding motion of $\Sigma$ in $\mathbb{R}^{N}$ and look what happens to its degree. It can change only when some of the $F_{\mathcal{R}}$ pass through 0 .

We divide our collection $\mathbf{R}$ of rainbow $r$-partitions into classes of an equivalence $\sim$, where $\mathcal{R} \sim \mathcal{R}^{\prime}$ if $\mathcal{R}^{\prime}$ can be obtained from $\mathcal{R}$ by permuting the $R_{i}$ and, if needed, moving $z$ back to the $r$ th class. For example,

$$
\mathcal{R}=\left(R_{1}, R_{2}, R_{3}\right) \sim \mathcal{R}^{\prime}=\left(R_{2}, R_{3} \backslash\{z\}, R_{1} \cup\{z\}\right) .
$$

Each class has $r$ ! members, and it turns out that, during the continuous motion, the simplices $F_{\mathcal{R}}$ for all $\mathcal{R}$ in the same class always pass through 0 simultaneously, and their contributions to the degree change by the same amount.

It follows that $\operatorname{deg}(\mathcal{C})$ may change only by multiples of $r$ ! during the motion. Since the degree for the special BMZ-collection $\mathcal{C}_{0}$ comes out as $D_{0}= \pm((r-1) !)^{d}$, the degree for every $\mathcal{C}$ is congruent to $D_{0}$ modulo $r$ !.

Here, finally, the primality of $r$ comes into play. When $r$ is a prime, and only then, we have $D_{0} \not \equiv 0(\bmod r !)$, and hence the degree is always nonzero as needed.

On the other hand, there are non-prime $r$ for which BMZ-collections $\mathcal{C}$ exist with degree 0 , so indeed the proof method breaks down (we suspect that this is the case for all non-prime $r$, but we have no proof at present). Of course, if the claim of the Blagojević-Matschke-Ziegler theorem failed for some (non-prime) $r$, one would have to look for a counterexample among the $\mathcal{C}$ with degree 0.

\section{The Sarkaria-Onn Transform}

We start filling out the details in the above outline. First we introduce the construction that assigns a point set in $\mathbb{R}^{N}$ to every rainbow $r$-partitions of a given BMZcollection. We present it in a slightly more general setting, ignoring the "rainbow" aspect.

We will use the notation $[k]=\{1,2, \ldots, k\}$ for a positive integer $k$.

For a point $x \in \mathbb{R}^{d}$ we write $x^{+}$for the vector $(x, 1) \in \mathbb{R}^{d+1}$ obtained by appending the component 1 to $x$.

\footnotetext{
${ }^{2}$ From the topological point of view, in this part we verify the well-known fact (cf. [5]) that the abstract simplicial complex underlying $\Sigma$ is an orientable pseudomanifold; this is a crucial part of the proof, as well as of the proofs in $[6,17]$.
} 
Fig. 5 The vectors $w_{1}, \ldots, w_{r}$ for $r=3$

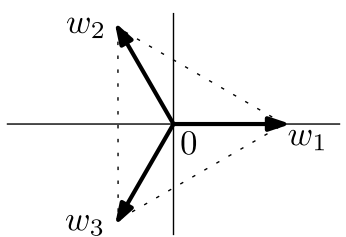

Let $w_{1}, \ldots, w_{r}$ be vectors in $\mathbb{R}^{r-1}$ forming the vertex set of a regular $(r-1)$ dimensional simplex with center at the origin; Fig. 5 illustrates the case $r=3$. We have $w_{1}+w_{2}+\cdots+w_{r}=0 .{ }^{3}$ Moreover, if $\alpha_{1}, \ldots, \alpha_{r}$ are real numbers with $\alpha_{1} w_{1}+$ $\cdots+\alpha_{r} w_{r}=0$, we have $\alpha_{1}=\alpha_{2}=\cdots=\alpha_{r}$, since every $r-1$ of the $w_{i}$ are linearly independent.

For $x \in \mathbb{R}^{d}$ and an index $i \in[r]$, we define a point

$$
\varphi_{i}(x):=x^{+} \otimes w_{i} \in \mathbb{R}^{N},
$$

called the ith clone of $x$. Here $N=(d+1)(r-1)$, and $\otimes$ stands for the (standard) tensor product: for arbitrary vectors $u \in \mathbb{R}^{m}$ and $v \in \mathbb{R}^{n}, u \otimes v$ is the vector

$$
\left(u_{1} v_{1}, u_{1} v_{2}, \ldots, u_{1} v_{n}, u_{2} v_{1}, u_{2} v_{2}, \ldots, u_{m} v_{n}\right) \in \mathbb{R}^{m n}
$$

Now let $\mathcal{P}=\left(P_{1}, P_{2}, \ldots, P_{r}\right)$ be an $r$-partition in $\mathbb{R}^{d}$, i.e., an $r$-tuple of pairwise disjoint finite sets in $\mathbb{R}^{d}$ (but the disjointness will be used only for a convenient notation; the claims below remain valid even if the $P_{i}$ may share points). Let $P=P_{1} \cup \cdots \cup P_{r}$ be the ground set.

We define the Sarkaria-Onn transform of $\mathcal{P}$ as the point set

$$
\Phi(\mathcal{P}):=\bigcup_{i=1}^{r}\left\{\varphi_{i}(p): p \in P_{i}\right\},
$$

and we let

$$
S_{\mathcal{P}}:=\operatorname{conv}(\Phi(\mathcal{P}))
$$

In words, for every point $p \in P_{i}$ we put the $i$ th clone of $p$ in $\Phi(\mathcal{P})$.

The following lemma is essentially from [14].

Lemma 3 (Sarkaria-Onn) Let $\mathcal{P}$ be an $r$-partition in $\mathbb{R}^{d}$. Then $\mathcal{P}$ has a Tverberg point, i.e., satisfies $\bigcap_{i=1}^{r} \operatorname{conv}\left(P_{i}\right) \neq \emptyset$, if and only if $0 \in S_{\mathcal{P}}$.

Proof For the reader's convenience, we sketch a proof; the omitted details are easy to fill in.

\footnotetext{
${ }^{3}$ The easiest way to see this to represent the regular $(r-1)$-simplex as the convex hull of the $r$ standard basis vectors in $\mathbb{R}^{r}$. Then we can identify $\mathbb{R}^{r-1}$ with the hyperplane $\left\{x \in \mathbb{R}^{r}: \sum_{i=1}^{r} x_{i}=1\right\}$, and choose a coordinate system such that the origin lies at the barycenter of the simplex, i.e., vector with all coordinates equal to $1 / r$. 
First, let us suppose that $x \in \bigcap_{i=1}^{r} \operatorname{conv}\left(P_{i}\right)$ is a Tverberg point. Thus, for every $i$ we can write $x=\sum_{p \in P_{i}} \xi_{p} p$ for some nonnegative reals $\xi_{p}$ with $\sum_{p \in P_{i}} \xi_{p}=1$. Then it is easy to check that

$$
0=\frac{1}{r} \sum_{i=1}^{r} \sum_{p \in P_{i}} \xi_{p} \varphi_{i}(p)
$$

holds, and that this expresses 0 as a convex combination of the points of $\Phi(\mathcal{P})$.

Conversely, let us suppose that $0 \in S_{\mathcal{P}}$. Thus, we can write

$$
0=\sum_{i=1}^{r} \sum_{p \in P_{i}} \alpha_{p}\left(p^{+} \otimes w_{i}\right)=\sum_{i=1}^{r}\left(\sum_{p \in P_{i}} \alpha_{p} p^{+}\right) \otimes w_{i}
$$

for some nonnegative $\alpha_{p}$ 's summing to 1 . Let $A_{i}:=\sum_{p \in P_{i}} \alpha_{p}$ and $s_{i}:=\sum_{p \in P_{i}} \alpha_{p} p$. By (1) we have $\sum_{i=1}^{r} A_{i} w_{i}=0$, and so, by the properties of the $w_{i}$, all the $A_{i}$ are equal to some $A$. Similarly, all the $s_{i}$ equal some $s \in \mathbb{R}^{d}$. Finally, one easily checks that $A>0$ (since not all of the $\alpha_{p}$ are 0 ) and that the point $\frac{1}{A} s$ is a Tverberg point.

In our considerations, we will need to interpret some other properties of $\Phi(\mathcal{P})$ in terms of $\mathcal{P}$. We recall that the affine hull aff(X) of a (finite) set $X \subseteq \mathbb{R}^{d}$ is the smallest affine subspace of $\mathbb{R}^{d}$ containing $X$. We also define the linear affine hull linaff $(X)$ as the translation of $\operatorname{aff}(X)$ to 0 , or in other words, as the set of all linear combinations $\sum_{i=1}^{n} \beta_{i} x_{i}$ with $x_{1}, \ldots, x_{n} \in X$ and $\sum_{i=1}^{n} \beta_{i}=0$.

Let us say that the partition $\mathcal{P}$ has an affine Tverberg point if $\bigcap_{i=1}^{r}$ aff $\left(P_{i}\right) \neq \emptyset$. Let us say that $\mathcal{P}$ has a Tverberg direction if $\bigcap_{i=1}^{r} \operatorname{linaff}\left(P_{i}\right) \neq\{0\}$; in other words, if there is a line parallel to each of the $\operatorname{aff}\left(P_{i}\right)$.

Lemma 4 For an $r$-partition $\mathcal{P}$ in $\mathbb{R}^{d}$, we have the following equivalences:

(i) $0 \in \operatorname{aff}(\Phi(\mathcal{P}))$ iff $\mathcal{P}$ has an affine Tverberg point.

(ii) The set $\Phi(\mathcal{P})$ is affinely dependent iff at least one of the $P_{i}$ 's is affinely dependent or $\mathcal{P}$ has a Tverberg direction.

Proof The proof is very similar to that of Lemma 3 and we only sketch it, leaving the details to the interested reader.

In (i), the assumption $0 \in \operatorname{aff}(\Phi(\mathcal{P}))$ can be written as $\sum_{p \in P} \alpha_{p} p=0$ for some $\alpha_{p}$ 's with $\sum_{p \in P} \alpha_{p}=1$. As in the proof of Lemma 3, $\sum_{p \in P} \alpha_{p} p=0$ implies that the sums $\sum_{p \in P_{i}} \alpha_{p}, i \in[r]$, are all equal to the same number $A$ and the sums $\sum_{p \in P_{i}} \alpha_{p} p$ are all equal to the same $s$. From $\sum_{p \in P} \alpha_{p} \neq 0$ we get $A \neq 0$, and thus $\frac{1}{A} s \in \operatorname{aff}\left(P_{i}\right)$ for all $i$. The reverse implication in (i) is proved by going through a very similar argument backwards.

As for (ii), we assume that the points of $\Phi(\mathcal{P})$ are affinely dependent, i.e., there exist reals $\alpha_{p}, p \in P$, summing to 0 and not all zero such that $\sum_{p \in P} \alpha_{p} p=0$. We again have $\sum_{p \in P_{i}} \alpha_{p}=A$ and $\sum_{p \in P_{i}} \alpha_{p} p=s$ for all $i \in[r]$. Since $\sum_{p \in P} \alpha_{p}=0$, we get $A=0$. If $s=0$, then at least one of the $P_{i}$ is affinely dependent, and otherwise, $s$ is a nonzero vector in $\bigcap_{i=1}^{r} \operatorname{linaff}\left(P_{i}\right)$. Again we omit the reverse implication. 


\section{Sufficiently General Position}

Some Conventions for BMZ-Collections Now we specialize to BMZ-collections. Let $\mathcal{C}=\left(C_{1}, \ldots, C_{d+2}\right)$ be a BMZ-collection, and let us write $C:=C_{1} \cup C_{2} \cup \cdots \cup$ $C_{d+2}$ for its ground set.

We also assume that the points of $C$ are numbered as $c_{1}, c_{2}, \ldots, c_{N+1}=z$, in such a way that $C_{1}$ consists of the first $r-1$ points $c_{1}, \ldots, c_{r-1}, C_{2}$ consists of the next $r-1$ points, etc.

Let $\mathcal{R}$ be a rainbow $r$-partition for $\mathcal{C}$. We define the combinatorial type of $\mathcal{R}$ as the set $\left\{(i, j): c_{j} \in R_{i}\right\} \subseteq[r] \times[N+1]$.

As in the proof outline, let $\mathbf{R}$ be the collection of all the maximal rainbow $r$ partitions for $\mathcal{C}$ having the point $z$ in the last class.

For $\mathcal{R}=\left(R_{1}, \ldots, R_{r}\right) \in \mathbf{R}$ and a point $a \in C$, we write $\mathcal{R}-a$ for the rainbow $r$-partition $\left(R_{1} \backslash\{a\}, \ldots, R_{r} \backslash\{a\}\right)$ (we remove $a$ from the class it belongs to).

For every $\mathcal{R} \in \mathbf{R}$, we have $z \in R_{r}$, and so each $\Phi(\mathcal{R})$ contains the point $z^{*}=\varphi_{r}(z)$. We set $F_{\mathcal{R}}:=\operatorname{conv}(\Phi(\mathcal{R}-z))$; if $S_{\mathcal{R}}$ is an $N$-dimensional simplex, which is usually the case, then $F_{\mathcal{R}}$ is the facet opposite to $z^{*}$ as in the outline.

Sufficiently General Position For defining the degree as sketched in the outline, we need that the simplices $F_{\mathcal{R}}$ are in a suitably general position. We adopt a "functional" approach, postulating the required properties in a definition.

We say that $\mathcal{C}$ is in a sufficiently general position if

- each $F_{\mathcal{R}}$ is an $(N-1)$-dimensional simplex, i.e., its vertices are affinely independent, and

- for every $\mathcal{R} \in \mathbf{R}$ and every $a \in C$ we have $0 \notin \operatorname{aff}(\Phi(\mathcal{R}-a))$; geometrically, the affine span of each facet of $S_{\mathcal{R}}$ avoids 0 .

It is easily seen that for $\mathcal{C}$ in sufficiently general position, the ray $\rho$ as in the outline (emanating from 0 in the direction opposite to $0 z^{*}$ ) is well defined and intersects each $F_{\mathcal{R}}$ in at most one point, which lies in the relative interior of $F_{\mathcal{R}}$.

Let $\mathcal{C}, \mathcal{C}^{\prime}$ be two BMZ-collections. We define their distance in the natural way, as $\max \left\{\left\|c_{i}-c_{i}^{\prime}\right\|: i=1,2, \ldots, N+1\right\}$ where $\|$.$\| is the Euclidean norm and c_{i}^{\prime}$ is, of course, the $i$ th point of $\mathcal{C}^{\prime}$.

We want to show that for every BMZ-collection $\mathcal{C}$, there are BMZ-collections $\mathcal{C}^{\prime}$ in sufficiently general position arbitrarily close to it. We proceed by a standard perturbation argument (an alternative route would be using points with algebraically independent coordinates in $\mathcal{C}^{\prime}$ ). This is a technical and somewhat tedious part (in the topological proof, it is taken care of by the general machinery, so one need not worry about it). Still, we prefer to include it, in order to make the proof complete.

Lemma 5 Let $\mathcal{C}$ be a BMZ-collection, and let $\varepsilon>0$ be given. Then there is a BMZcollection $\mathcal{C}^{\prime}$ in sufficiently general position at distance at most $\varepsilon$ from $\mathcal{C}$.

Proof First we observe that for $\mathcal{R} \in \mathbf{R}$, since the classes $R_{1}, \ldots, R_{r}$ are rainbow, each of the classes $R_{i}$ has at most $d+1$ points, except possibly for $R_{r}$, which may contain up to $d+2$ points. 
According to Lemma 4, the conditions in the definition of sufficiently general position of $\mathcal{C}$ are implied by the following:

(i) Every at most $d+1$ points of $C$ are affinely independent.

(ii) For every $\mathcal{R} \in \mathbf{R}$, the partition $\mathcal{R}-z$ has no Tverberg direction.

(iii) For every $\mathcal{R} \in \mathbf{R}$ and every $a \in C$, the partition $\mathcal{R}-a$ has no affine Tverberg point.

To seasoned geometers, (i)-(iii) are probably obvious by codimension count. Still, we include a more detailed argument.

We first recall a perturbation argument for achieving (i), where it is entirely simple and standard. Condition (i) is a conjunction of $\left(\begin{array}{c}|C| \\ d+1\end{array}\right)$ requirements of the form "the points in $C_{I}:=\left\{c_{i}: i \in I\right\}$ are affinely independent", where $I$ runs through all $(d+$ 1)-element subsets of $C$. We enumerate all such $I$ as $I_{1}, I_{2}, \ldots$ and we deal with them one by one.

First we consider $I_{1}$; say that $I_{1}=\{1,2, \ldots, d+1\}$. The one-point set $\left\{c_{1}\right\}$ is affinely independent, of course, and so is $\left\{c_{1}, c_{2}\right\}$, assuming that the points of $C$ are all distinct. Next, it is clear that we can move $c_{3}$ by at most $\frac{\varepsilon}{2}$ so that $C_{3}:=\left\{c_{1}, c_{2}, c_{3}\right\}$ is affinely independent, too. Then we successively move $c_{4}, \ldots, c_{d+1}$, each by at most $\frac{\varepsilon}{2}$, and we make $C_{I_{1}}$ affinely independent. Moreover, crucially, there exists some $\varepsilon_{1}>0$ such that if we move the points of $C_{I_{1}}$ arbitrarily by at most $\varepsilon_{1}$, then $C_{I_{1}}$ remains affinely independent. Using this $\varepsilon_{1}$, we make $C_{I_{2}}$ affinely independent, obtaining some even much smaller $\varepsilon_{2}>0$, etc., until all the index sets $I_{j}$ have been exhausted.

A similar procedure can be applied to achieve (ii) and (iii). For example, in (iii), we fix $\mathcal{R}$ and $a$ and see how can we make sure that $\mathcal{R}-a$ has no affine Tverberg point.

Let us write $R_{i}^{-}:=R_{i} \backslash\{a\}$. Each of the subspaces $L_{i}:=\operatorname{aff}\left(R_{i}\right)$ has dimension at most $\left|R_{i}^{-}\right|-1$.

In general, if two affine subspaces $K, L \subset \mathbb{R}^{d}$ of dimensions $k, \ell$, respectively, are in general position, we have $\operatorname{dim}(K \cap L)=\max (-1, k+\ell-d)$, where dimension -1 means empty intersection. Thus, we can move $L_{2}, L_{3}, \ldots, L_{r}$ one by one (by moving the points of the $\left.R_{i}^{-}\right)$, inductively achieving $\operatorname{dim}\left(L_{1} \cap \cdots \cap L_{i}\right)=$ $\max \left(-1,\left(\sum_{j=1}^{i}\left|R_{j}^{-}\right|\right)-i-(i-1) d\right)$. Since $\sum_{j=1}^{r}\left|R_{j}^{-}\right|=N=(r-1)(d+1)$, we get $\operatorname{dim}\left(L_{1} \cap \cdots \cap L_{r}\right)=-1$, which means no affine Tverberg point.

Condition (ii) is achieved with a very similar dimension-counting, which we omit.

A Remark on Degenerate $S_{\mathcal{R}}$ 's The "exceptional" $S_{\mathcal{R}}$ 's that are only $(N-1)$ dimensional, even for $\mathcal{C}$ in sufficiently general position, are obtained for the $\mathcal{R}$ with the last class $R_{r}$ of size $d+2$. Then $R_{r}$ cannot be affinely independent, and thus (by Lemma 4 ) the vertex set of $S_{\mathcal{R}}$ is not affinely independent - the point $z^{*}$ is contained in the affine span of $F_{\mathcal{R}}$. But this does not matter since, for $\mathcal{C}$ in sufficiently general position, the affine span of $F_{\mathcal{R}}$ avoids 0 and thus such an $F_{\mathcal{R}}$ cannot influence the degree. (Or in other words, such a partition $\mathcal{R}$ is never Tverberg for $\mathcal{C}$ in sufficiently general position.) 
Continuous Motion of $\mathcal{C}$ Later on, in the continuous motion argument, we will need to consider two BMZ-collections $\mathcal{C}, \mathcal{C}^{\prime}$ and analyze what happens with the degree when we continuously move the points, starting from $\mathcal{C}$ and ending at $\mathcal{C}^{\prime}$.

As we will see, the moving collection can be kept in sufficiently general position all the time except for finitely many critical times.

We will also need some control over what happens at the critical times. Let $\mathcal{R} \in \mathbf{R}$ and let $a \in C, a \neq z$. We call the set $G:=\operatorname{conv}\left(\Phi(\mathcal{R}-z-a)\right.$ ) a ridge (if $S_{\mathcal{R}}$ is an $N$-simplex, which is typically the case, then $G$ is a facet of $F_{\mathcal{R}}$ and thus a ridge of $S_{\mathcal{R}}$ ). We say that $\mathcal{C}$ is in almost general position if all ridges avoid 0.

Lemma 6 Let $\mathcal{C}, \mathcal{C}^{\prime}$ be BMZ-collections in sufficiently general position. Then there is a continuous family $\mathcal{C}^{(t)}$ of BMZ-collections, $t \in[0,1]$, such that $\mathcal{C}^{(0)}=\mathcal{C}, \mathcal{C}^{(1)}=\mathcal{C}^{\prime}$, each $\mathcal{C}^{(t)}$ is in almost general position, and there is a finite set $T \subset[0,1]$ of critical times such that $\mathcal{C}^{(t)}$ is in sufficiently general position for all $t \notin T$.

Proof For simplicity, we move one point at a time. It suffices to establish the lemma for $\mathcal{C}, \mathcal{C}^{\prime}$ such that $c_{i}=c_{i}^{\prime}$ for all $i \neq 1$. Moreover, since all BMZ-collections sufficiently close to $\mathcal{C}^{\prime}$ are also in a sufficiently general position, it is enough that we can move $c_{1}$ to any position $c_{1}^{\prime \prime}$ sufficiently close to $c_{1}^{\prime}$, in a way satisfying the conclusion of the lemma, since then the motion from $c_{1}^{\prime \prime}$ to $c_{1}^{\prime}$ is for free.

Thus, from now on we assume that $c_{1}$ moves to $c_{1}^{\prime \prime}$ along a segment at uniform speed, while all the other points are stationary. Let $c_{1}^{(t)}$ be the position of the moving point at time $t$.

First we check that there are only finitely many times where $\mathcal{C}^{(t)}$ is not in sufficiently general position. We need to consider conditions (i)-(iii) from the proof of Lemma 5. For the sake of illustration, we check (iii), leaving the rest to the reader.

Still referring to that proof, we consider the affine subspaces $L_{1}, \ldots, L_{r}$ (for a particular $\mathcal{R}$ and $a$ ). We renumber them so that the moving point is among those defining $L_{r}$, so $L_{1}, \ldots, L_{r-1}$ are stationary and $L_{r}^{(t)}$ is moving. Let $\bar{L}_{r}:=\bigcup_{t \in[0,1]} L_{r}^{(t)}$; since $c_{1}^{(t)}$ traces a segment, $\bar{L}_{r}$ is contained in the affine span of $L_{r} \cup\left\{c_{1}^{\prime \prime}\right\}$, which is an affine subspace of dimension $\operatorname{dim}\left(L_{r}\right)+1$. By sufficiently general position of $\mathcal{C}$ we know that $\operatorname{dim}\left(L_{1} \cap \cdots \cap L_{r-1}\right)+\operatorname{dim}\left(L_{r}\right)<d$, and thus, by altering the position of $c_{1}^{\prime \prime}$ by an arbitrarily small amount, we can achieve that $L_{1} \cap \cdots \cap L_{r-1}$ meets $\bar{L}_{r}$ in at most one point. This adds at most one critical time.

It remains to check that $\mathcal{C}^{(t)}$ is always in almost general position, for which the argument is very similar to the previous one. We want that all ridges avoid 0 all the time. We strengthen the condition to the affine span of all ridges avoiding 0, which translates into $\mathcal{R}-z-a$ never having an affine Tverberg point. Thus, we again deal with affine subspaces $L_{1}, \ldots, L_{r}$; we again assume that $L_{1}, \ldots, L_{r-1}$ are stationary and $L_{r}^{(t)}$ is moving, and let $\bar{L}_{r}$ be the set traced by $L_{r}^{(t)}$ during the motion, contained in an affine subspace of dimension $\operatorname{dim}\left(L_{r}\right)+1$. However, compared to the previous argument, now the sum of the dimensions of the $L_{i}$ is one smaller, and this allows us to achieve $L_{1} \cap \cdots \cap L_{r-1} \cap \bar{L}_{r}=\emptyset$, again by changing the position of $c_{1}^{\prime \prime}$ by an arbitrarily small amount. 


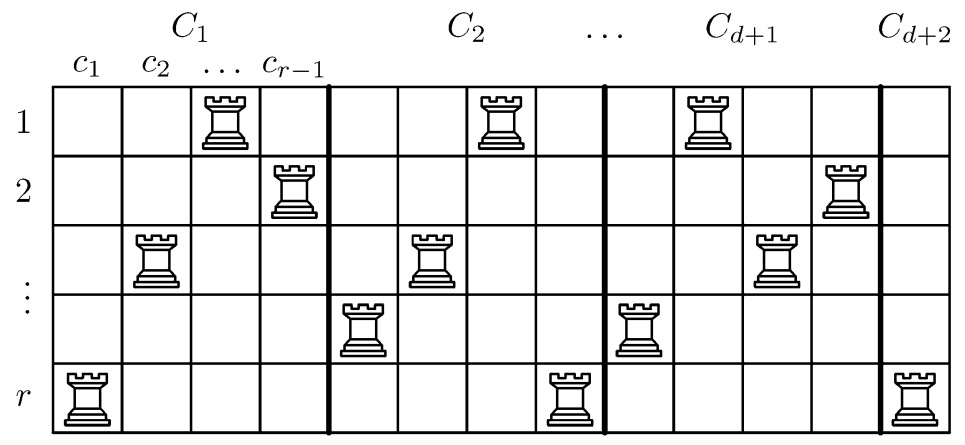

Fig. 6 The combinatorial type of a rainbow $r$-partition represented by a non-attacking placement of rooks on chessboards

Sufficiently General Position May be Assumed We will prove Theorem 2 with the additional assumption that $\mathcal{C}$ is in sufficiently general position. By Lemma 5, each BMZ-collection can be approximated by such BMZ-collections arbitrarily closely, and thus the validity of Theorem 2 for an arbitrary $\mathcal{C}$ follows by a routine limiting argument, which we omit (see, for example, [15, Lemma 2] for a very similar one).

\section{The Degree}

For every $(N-1)$-dimensional simplex $F_{\mathcal{R}}$, we now define a $\operatorname{sign} \operatorname{sgn}\left(F_{\mathcal{R}}\right)$ (often we also write just $\operatorname{sgn}(\mathcal{R})$, since $F_{\mathcal{R}}$ is fully determined by $\mathcal{R}$ ). In the language introduced in the proof outline, the sign +1 means that the side of $F_{R}$ visible from 0 is negative, and -1 means that it is positive.

The sign is the product of two factors, which we call the geometric sign $\operatorname{gsgn}(\mathcal{R})$ and the combinatorial sign $\operatorname{csgn}(\mathcal{R})$.

The geometric sign is easy to define. We set up the $N \times N$ matrix $M$ with the coordinates of the $i$ th vertex of $F_{\mathcal{R}}$ (we recall that the points in the ground set $C$ are numbered as $c_{1}, \ldots, c_{N+1}$, which induces a linear ordering of the vertices of $F_{\mathcal{R}}$ ), and we put

$$
\operatorname{gsgn}(\mathcal{R}):=\operatorname{sgn} \operatorname{det}(M) .
$$

The combinatorial sign is slightly more complicated. We recall that the combinatorial type of $\mathcal{R}$ is the set $\left\{(i, j): c_{j} \in R_{i}\right\} \subseteq[r] \times[N+1]$. It can be depicted using an $r \times(N+1)$ array of squares, whose $i$ th row corresponds to the sets $R_{i}$ of $\mathcal{R}$ and whose $j$ th column corresponds to the $j$ th point of $C$; see Fig. 6 . Then we place a rook (chess figure) to each square $(i, j)$ with $c_{j} \in R_{i}$.

Let us think of the array as $d+1$ chessboards, each with $r$ rows and $r-1$ columns, placed side by side, plus one "degenerate" $r \times 1$ chessboard on the right. Then the maximal rainbow $r$-partitions exactly correspond to maximal placements of mutually non-attacking rooks on each of the chessboards (in particular, each of the $r \times(r-1)$ chessboards has $r-1$ rooks on it). The condition that $z \in R_{r}$ then says that the last narrow chessboard should have the rook in the last row. 
The vertices of $F_{\mathcal{R}}$ correspond to the rooks in the first $d+1$ chessboards. The placement of the $r-1$ rooks on the $k$ th chessboard defines a permutation $\pi_{k}$ of $[r]$; namely, for $j \leq r-1, \pi_{k}(j)$ is the index of the row containing the rook of the $i$ th column, and $\pi_{k}(r)$ is the index of the unique row with no rook.

The combinatorial sign of $\mathcal{R}$ is defined as

$$
\operatorname{csgn}(\mathcal{R}):=\prod_{k=1}^{d+1} \operatorname{sgn} \pi_{k} .
$$

The Degree As in the outline, we define

$$
\Sigma=\Sigma(\mathcal{C}):=\bigcup_{\mathcal{R} \in \mathbf{R}} F_{\mathcal{R}}
$$

and the degree of $\Sigma$ is

$$
\operatorname{deg}(\Sigma):=\sum_{\mathcal{R} \in \mathbf{R}: \rho \cap F_{\mathcal{R}} \neq \emptyset} \operatorname{sgn}\left(F_{\mathcal{R}}\right),
$$

where $\operatorname{sgn}\left(F_{\mathcal{R}}\right)=\operatorname{sgn}(\mathcal{R})=\operatorname{gsgn}(\mathcal{R}) \operatorname{csgn}(\mathcal{R})$. In other words, the degree is the sum of $\operatorname{sgn}\left(F_{\mathcal{R}}\right)$ over all $\mathcal{R} \in \mathbf{R}$ that are Tverberg. Since $\Sigma$ is determined by $\mathcal{C}$, we will also write $\operatorname{deg}(\mathcal{C})$ instead of $\operatorname{deg}(\Sigma)$.

\section{The Continuous Motion Argument}

Here we prove the promised invariance of the degree modulo $r$ !.

Proposition 7 If $\mathcal{C}$ and $\mathcal{C}^{\prime}$ are two BMZ-collections (for the same $d$ and $r$ ), both in sufficiently general position, then

$$
\operatorname{deg}(\mathcal{C}) \equiv \operatorname{deg}\left(\mathcal{C}^{\prime}\right)(\bmod r !)
$$

First we want to verify that the simplices $F_{\mathcal{R}}$ are "glued together" properly. Let us call the $(N-2)$-dimensional faces of $F_{\mathcal{R}}$ the ridges.

Lemma 8 Let $G$ be a ridge of some $F_{\mathcal{R}}$. Then there is exactly one $\mathcal{R}^{\prime} \in \mathbf{R}$ distinct from $\mathcal{R}$ having $G$ as a ridge, and we have $\operatorname{csgn}\left(\mathcal{R}^{\prime}\right)=-\operatorname{csgn}(\mathcal{R})$. (In topological terminology, this is the "orientable pseudomanifold" property.)

Proof This is easy to see using the rook interpretation. The simplex $F_{\mathcal{R}}$ corresponds to a maximal placement of rooks on the first $d+1$ chessboards, and $G$ is obtained by removing one of the rooks, say from the $k$ th chessboard. Now the $k$ th chessboard has one empty column and two empty rows, so there are two possibilities of putting the rook back-one corresponding to $F_{\mathcal{R}}$, and the other to $F_{\mathcal{R}^{\prime}}$.

The permutation $\pi_{k}$ for $\mathcal{R}$ and the one for $\mathcal{R}^{\prime}$ differ by a single transposition, and $\operatorname{so} \operatorname{csgn}(\mathcal{R})=-\operatorname{csgn}\left(\mathcal{R}^{\prime}\right)$ as claimed. 
Fig. 7 The moving ray crossing a ridge

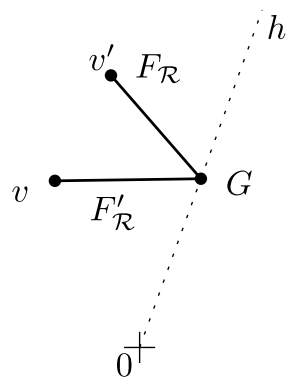

$h$

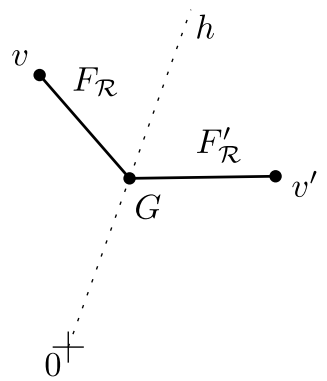

Next, we want to see that the degree of $\Sigma$ can be computed with respect to an arbitrary (generic) ray. Let $\mathcal{C}$ be a BMZ-collection, exceptionally assumed to be only in almost general position (which, as we recall, means that all the ridges of the $F_{\mathcal{R}}$ 's avoid the origin).

Let $\psi$ be a ray in $\mathcal{R}^{N}$ emanating from 0 . We call $\psi$ generic for $\mathcal{C}$ if it does not intersect any ridge. It follows that if such a generic $\psi$ intersects some $F_{\mathcal{R}}$, then $F_{\mathcal{R}}$ must be an $(N-1)$-dimensional simplex and $\psi$ intersects it in a single point lying in the relative interior of $F_{\mathcal{R}}$.

Clearly, almost all rays (in the sense of measure) are generic. Moreover, if $\psi$ is generic for some $\mathcal{C}$, then it is also generic for all $\mathcal{C}^{\prime}$ sufficiently close to $\mathcal{C}$; this will be useful later on.

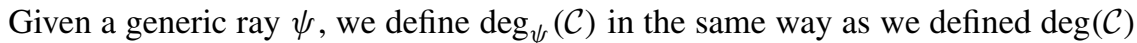
using $\rho$; that is, as $\sum_{\mathcal{R} \in \mathbf{R}: \psi \cap F_{\mathcal{R}} \neq \emptyset} \operatorname{sgn}(\mathcal{R})$.

Lemma 9 Let $\mathcal{C}$ be a BMZ-collection in sufficiently general position. If $\psi$ and $v$ are generic rays for $\mathcal{C}$, then $\operatorname{deg}_{\psi}(\mathcal{C})=\operatorname{deg}_{v}(\mathcal{C})$.

Proof We can continuously move $\psi$ to $v$ so that it remains generic all the time, except for finitely many moments where it intersects some ridge (or perhaps several ridges) at an interior point. So it suffices to check that the degree cannot change by crossing a ridge $G$.

As we know from Lemma 8, the ridge $G$ is shared by exactly two facets $F_{\mathcal{R}}$ and $F_{\mathcal{R}^{\prime}}$, with $\operatorname{csgn}(\mathcal{R})=-\operatorname{csgn}\left(\mathcal{R}^{\prime}\right)$. Let $v$ be the vertex of $F_{\mathcal{R}}$ not in $G$, and similarly for $v^{\prime}$ and $F_{\mathcal{R}^{\prime}}$. As we saw in the proof of Lemma 8, $v$ and $v^{\prime}$ are two different clones of the same point $c_{j} \in C$.

Let $h$ be the hyperplane spanned by $G$ and 0 . First let us suppose that both $v$ and $v^{\prime}$ are on the same side of $h$ (Fig. 7 left). Then the moving ray intersects both of $F_{\mathcal{R}}, F_{\mathcal{R}^{\prime}}$ before crossing $G$ and none of them after the crossing, or the other way around.

Let $M$ and $M^{\prime}$ be the matrices used in the definition of the geometric signs of $F_{\mathcal{R}}$ and $F_{\mathcal{R}^{\prime}}$, respectively. They differ in a single row, which is $v$ in $M$ and $v^{\prime}$ in $M^{\prime}$ (the row is in the same position since $v$ and $v^{\prime}$ are both clones of $c_{j}$ ). Since $v$ and $v^{\prime}$ are on the same side of $h$, we have $\operatorname{sgn}(\operatorname{det} M)=\operatorname{sgn}\left(\operatorname{det} M^{\prime}\right)$, and thus $F_{\mathcal{R}}$ and $F_{\mathcal{R}^{\prime}}$ have the same geometric signs.

Altogether we get $\operatorname{sgn}(\mathcal{R})=-\operatorname{sgn}\left(\mathcal{R}^{\prime}\right)$, and thus when the ray intersects both of $F_{\mathcal{R}}, F_{\mathcal{R}^{\prime}}$, their contributions to the degree cancel out. By a similar argument, which 
we omit, one gets that in the other case, as in Fig. 7 right, $\operatorname{sgn}(\mathcal{R})=\operatorname{sgn}\left(\mathcal{R}^{\prime}\right)$, and so in both cases the degree remains constant.

Let $\mathcal{R} \in \mathbf{R}$ be a rainbow $r$-partition of a BMZ-collection $\mathcal{C}$ (in sufficiently general position). For a permutation $\pi$ of $[r]$, let $\mathcal{R}^{\pi}$ be the rainbow $r$-partition obtained by permuting the classes of $\mathcal{R}$ according to $\pi$ and moving $z$ back to the last class:

$$
\mathcal{R}^{\pi}:=\left(R_{\pi(1)} \backslash\{z\}, R_{\pi(2)} \backslash\{z\}, \ldots, R_{\pi(r-1)} \backslash\{z\}, R_{\pi(r)} \cup\{z\}\right) .
$$

We need to understand how the combinatorial and geometric signs of $\mathbb{R}^{\pi}$ are related to those of $\mathcal{R}$.

Lemma 10 For $\mathcal{R}$ and $\mathcal{R}^{\pi}$ as above, we have

$$
\operatorname{csgn}\left(\mathcal{R}^{\pi}\right)=\operatorname{sgn}(\pi)^{d+1} \operatorname{csgn}(\mathcal{R}), \quad \operatorname{gsgn}\left(\mathcal{R}^{\pi}\right)=\operatorname{sgn}(\pi)^{d+1} \operatorname{gsgn}(\mathcal{R}) .
$$

Proof In the representation of $\mathcal{R}$ with rooks, passing to $\mathcal{R}^{\pi}$ means that we permute the rows of the first $d+1$ chessboards. From this we immediately get the first relation, $\operatorname{csgn}\left(\mathcal{R}^{\pi}\right)=\operatorname{sgn}(\pi)^{d+1} \operatorname{csgn}(\mathcal{R})$.

For the geometric sign, it suffices to consider the case where $\pi$ is a transposition exchanging two indices $i, j$ and show that the geometric sign changes by the factor of $(-1)^{d+1}$ (an arbitrary $\pi$ can be expressed as a composition of such transpositions).

The effect of such a transposition on the vertex set of $F_{\mathcal{R}}$ is that the $i$ th clones of the points of $R_{i}$ are replaced with the $j$ th clones, and the reverse happens for the points of $R_{j}$ (ignoring $z$ ).

Let $M$ be the matrix as in the definition of $\operatorname{gsgn}(\mathcal{R})$, and let $M^{\pi}$ be the one for $\operatorname{gsgn}\left(\mathcal{R}^{\pi}\right)$. Thus, a row of the form $x^{+} \otimes w_{i}$ in $M$ is replaced by $x^{+} \otimes w_{j}$ in $M^{\pi}$. Similarly, $x^{+} \otimes w_{j}$ is replaced by $x^{+} \otimes w_{i}$, and all other rows remain unchanged.

Now we use the choice of the vectors $w_{1}, \ldots, w_{r}$. They form the vertex set of a regular simplex, and so there is a linear map $f: \mathbb{R}^{r-1} \rightarrow \mathbb{R}^{r-1}$ that interchanges $w_{i}$ with $w_{j}$ and leaves all the other $w_{k}$ fixed (namely, $f$ is a suitable mirror reflection).

Let $A$ be the matrix of $f$ with respect to the standard basis of $\mathbb{R}^{r-1}$. Then we can write $M^{\pi}=B M$, where $B$ is the block-diagonal matrix with $d+1$ blocks $A$ on the diagonal. Thus, $\operatorname{det}\left(M^{\pi}\right)=\operatorname{det}(A)^{d+1} \operatorname{det}(M)$, and since $f$ is a mirror reflection, and thus orientation-reversing, we have $\operatorname{sgn}(\operatorname{det} A)=-1$. So the geometric sign changes by $(-1)^{d+1}$ as claimed.

Proof of Proposition 7 The main trick in the proof is to alternate moving the ray and the points, thereby avoiding "too degenerate" situations.

Using Lemma 6, we may assume that $\mathcal{C}$ and $\mathcal{C}^{\prime}$ are connected by a continuous family $\mathcal{C}^{(t)}$. Each $\mathcal{C}^{(t)}$ is in almost general position, and it is in sufficiently general position except for finitely many critical times.

For every $t \in[0,1]$, including critical ones, we can choose a generic ray for $\mathcal{C}^{(t)}$, which also remains generic for all $\mathcal{C}^{\left(t^{\prime}\right)}$ with $t^{\prime}$ in some open interval around $t$. By compactness, the interval $[0,1]$ can be covered by finitely many of these open intervals $I_{1}, \ldots, I_{m}$, each of them corresponding to some generic ray $\psi_{i}$. 
By Lemma 9, on the overlapping part $I_{i} \cap I_{j}$ we can "measure" the degree using either $\psi_{i}$ or $\psi_{j}$ with the same result. Therefore, it suffices to show that if $I \subseteq[0,1]$ is an interval such that $\psi$ is a generic ray for all $\mathcal{C}^{(t)}$ with $t \in I$, then $\operatorname{deg}_{\psi}\left(\mathcal{C}^{(t)}\right)$ may change only by multiples of $r$ !.

The degree may change only at critical values of $t$; let $t_{0} \in I$ be one of the critical values. Let us see how the contribution of some $F_{\mathcal{R}}$ to $\operatorname{deg}_{\psi}\left(\mathcal{C}^{(t)}\right)$ may change at $t_{0}$. (More formally, we should write $F_{\mathcal{R}^{(t)}}$ instead of $F_{\mathcal{R}}$, where $\mathcal{R}^{(t)}$ is a rainbow $r$ partition of $\mathcal{C}^{(t)}$ whose combinatorial type does not depend on $t$. But we drop the superscript, keeping the dependence on $t$ implicit.)

A necessary condition for the change is that $F_{\mathcal{R}}$ intersects $\psi$ just before or just after $t_{0}$. If it intersects $\psi$ both just before and just after $t_{0}$, then, using the genericity of $\psi$, one can see that the geometric sign of $F_{\mathcal{R}}$ does not change, and so its contribution to the degree does not change either. Thus, the only possibility is that $F_{\mathcal{R}}$ intersects $\psi$ just before $t_{0}$ and does not intersect it just after, or the other way round.

By symmetry, it suffices to consider only the first case. Let us also assume that $\operatorname{sgn}(\mathcal{R})=+1$ for $t<t_{0}$ (in some small open interval ending in $t_{0}$, that is). Then, since $F_{\mathcal{R}}$ stopped intersecting $\psi$ at $t_{0}$, it must have passed 0 , and therefore, its geometric sign changed. Thus, $\operatorname{sgn}(\mathcal{R})=-1$ for $t>t_{0}$, and the contribution of $F_{\mathcal{R}}$ to $\operatorname{deg}\left(\mathcal{C}^{(t)}\right)$ has decreased by 1 at $t_{0}$.

Now we consider a permutation $\pi$ of $[r]$ and the rainbow $r$-partition $\mathcal{R}^{\pi}$, again depending on $t$. By Lemma 10 , we have $\operatorname{sgn}\left(\mathcal{R}^{\pi}\right)=\operatorname{sgn}(\mathcal{R})$ all the time, $\operatorname{so} \operatorname{sgn}\left(\mathcal{R}^{\pi}\right)$ also changes from +1 to -1 at $t_{0}$. Since the geometric sign of $F_{\mathcal{R}^{\pi}}$ changes at $t_{0}$ (again by Lemma 10), it means that $F_{\mathcal{R}^{\pi}}$ passed through 0 at $t_{0}$. So either it intersected $\psi$ just before $t_{0}$ and it does not intersect it just after, or vice versa. In both cases, the contribution of $F_{\mathcal{R}^{\pi}}$ to $\operatorname{deg}\left(\mathcal{C}^{(t)}\right)$ has also decreased by 1 at $t_{0}$.

Since there are $r$ ! choices for $\pi$, it follows that the degree may change only by multiples of $r$ ! as claimed. The proposition is proved.

\section{Computing the Degree of a Special BMZ-Collection}

Here is the last step in the proof of Theorem 2.

Lemma 11 There is a BMZ-collection $\mathcal{C}_{0}$ in sufficiently general position such that

$$
\left|\operatorname{deg} \mathcal{C}_{0}\right|=((r-1) !)^{d+1}
$$

Proof The first $d+1$ color classes of $\mathcal{C}_{0}$ are small clusters around the vertices of a regular $d$-dimensional simplex, as in Fig. 2, and the single point $z$ of the last class is placed at the center of gravity of that simplex.

It is easy to see (and well known) that the Tverberg rainbow $r$-partitions $\mathcal{R}$ of $\mathcal{C}_{0}$ with $\mathcal{R} \in \mathbf{R}$ have $R_{r}=\{z\}$, and the other $R_{i}$ each use exactly one point of each $C_{j}$, $j=1,2, \ldots, d+1$. In the rook interpretation, they correspond to rook placements where the $r$ th row contains only the single rook in the last column, and from this one immediately gets that their number is $((r-1) !)^{d+1}$.

It remains to see that all of these Tverberg $\mathcal{R}$ 's have the same sign. It suffices to consider the effect of a local change, where we swap two adjacent rows in one of 
the first $d+1$ chessboards (which corresponds to moving some $c_{j} \in C_{k}$ from $R_{i}$ to $R_{i+1}$ and some $c_{j^{\prime}} \in C_{k}$ from $R_{i+1}$ to $R_{i}, i+1 \leq r-1$ ). This obviously changes the combinatorial sign.

It remains to show that the geometric sign is also changed by the swap. Let $\mathcal{R}$ be the Tverberg $r$-partition before the swap and $\mathcal{R}^{\leftrightarrow}$ the one after the swap, and let $M$ and $M^{\leftrightarrow}$ be the corresponding matrices for $F_{\mathcal{R}}$ and $F_{R \leftrightarrow}$, as in the definition of the geometric sign. Thus, the $j$ th row is $\varphi_{i}\left(c_{j}\right)$ in $M$ and $\varphi_{i+1}\left(c_{j}\right)$ in $M^{\leftrightarrow}$, and the $j^{\prime}$ th row is $\varphi_{i+1}\left(c_{j^{\prime}}\right)$ in $M$ and $\varphi_{i}\left(c_{j^{\prime}}\right)$ in $M^{\leftrightarrow}$.

Let $M^{\prime}$ denote the matrix obtained from $M^{\leftrightarrow}$ by interchanging the $j$ th row with the $j^{\prime}$ th row. We have $\operatorname{det}\left(M^{\prime}\right)=-\operatorname{det}\left(M^{\leftrightarrow}\right)$, and we want to check that $\operatorname{sgn} \operatorname{det}\left(M^{\prime}\right)=$ sgn $\operatorname{det}(M)$.

We can regard $M^{\prime}$ as the matrix of vertex coordinates for the $(N-1)$-dimensional simplex $F_{\mathcal{R}}$ for a different $\mathrm{BMZ}$-collection $\mathcal{C}_{0}^{\prime}$, namely, the one obtained from $\mathcal{C}_{0}$ by interchanging $c_{j}$ with $c_{j^{\prime}}$. We prove a more general statement: whenever $\mathcal{C}_{0}^{\prime}$ is a BMZ-collection obtained from $\mathcal{C}_{0}$ by moving each of the points $c_{j}$ within its cluster arbitrarily (and keeping $z$ fixed), then $\operatorname{sgn} \operatorname{det}\left(M^{\prime}\right)=\operatorname{sgn} \operatorname{det}(M)$.

It suffices to check that during a continuous motion of some $c_{j}$ within its cluster, sgn $\operatorname{det}(M)$ remains constant. This sign may change only when the simplex $F_{\mathcal{R}}$ becomes degenerate, or when the hyperplane spanned by $F_{\mathcal{R}}$ passes through 0 .

These two conditions translate, according to Lemma 4, to the following: during the continuous motion, the points of each class $R_{i}, i<r$, remain affinely independent, and the $r$-partition $\mathcal{R}-z$ never has either an affine Tverberg point or a Tverberg direction. The former holds because each $R_{i}$ has one point in each cluster. The latter holds trivially since the $r$ th class of $\mathcal{R}-z$ is empty. This concludes the proof of Lemma 11.

Now we have completed all steps from the proof outline, and thus Theorem 2 is proved.

\section{Conclusion}

Configurations with Degree 0 Suppose that there is an integer $r$ for which there exists a BMZ-collection without a Tverberg point. Then the degree of this collection has to be 0 , and thus $r$ cannot be a prime number.

We performed computational experiments in the case $r=4$, with $d=2,3$. We generated BMZ-collections at random inside the unit square (or cube). We frequently obtained collections with degree 0 ; however, all of them had a Tverberg point. See Fig. 8 for a configuration with degree zero and few Tverberg partitions. We also obtained a collection of degree 0 for $r=6$ and $d=2$. In this case the computation was already quite time consuming (with our algorithm), and thus we performed only a small number of experiments.

We believe that BMZ-collections of degree 0 exist for all non-prime $r$ and in all dimensions, but unfortunately, we do not have a proof for this.

A natural idea for such a proof is to start with two BMZ-collections $\mathcal{C}_{1}$ and $\mathcal{C}_{2}$, one of a positive degree and one of a negative degree, and then transform $\mathcal{C}_{1}$ to $\mathcal{C}_{2}$ 

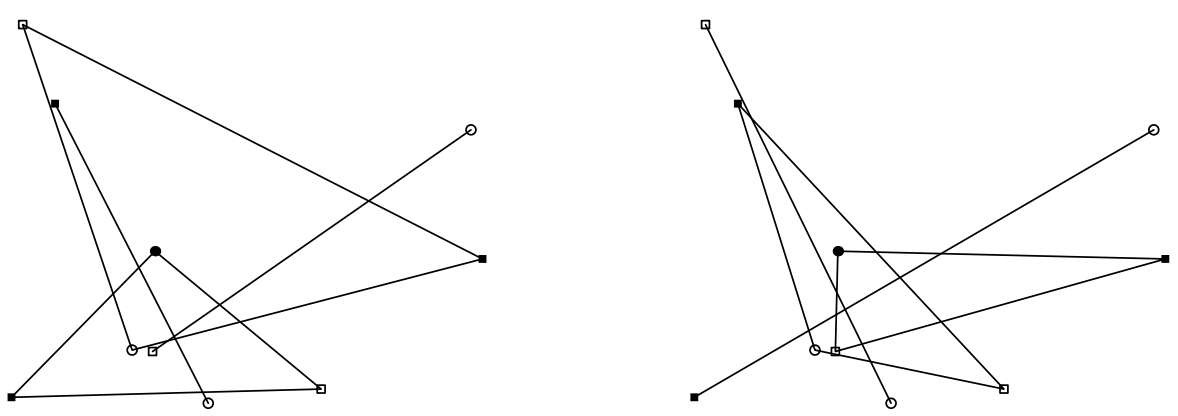

Fig. 8 A BMZ-collection with $r=4$ and $d=2$ of degree zero with only two different Tverberg partitions (more precisely with only two equivalence classes of $\sim$ )

by a (generic) continuous motion. If we knew that the degree may jump only by $\pm r$ ! during the motion, we would reach degree 0 at some moment (since the degree is always congruent to $((r-1) !)^{d}$ modulo $r$ !, as we know, and $((r-1) !)^{d}$ is divisible by $r$ ! for $d \geq 2$ and non-prime $r$ ). However, it turns out that even during a generic motion, there may be jumps by larger multiples of $r$ !, and so a subtler argument is needed.

A Direct Definition of Sign? A natural question is, whether one can define the sign of a rainbow partition directly, without going through the Sarkaria-Onn transform. However, it seems that if there is such a direct definition (only referring to the mutual position of the points of the rainbow partition) at all, it has to be rather complicated. We will illustrate this with an example concerning the simplest nontrivial case, with $d=2$ and $r=3$.

Thus, we consider points $c_{1}, c_{2}, \ldots, c_{6}, z$ in the plane, and the following BMZcollection: $C_{1}=\left\{c_{1}, c_{2}\right\}, C_{2}=\left\{c_{3}, c_{4}\right\}, C_{3}=\left\{c_{5}, c_{6}\right\}, C_{4}=\{z\}$. We consider several rainbow partitions $\mathcal{R} \in \mathbf{R}$ and the dependence of $\operatorname{sgn}(\mathcal{R})$ on the positions of the $c_{i}$. From the definition of the sign we get $\operatorname{sgn}(\mathcal{R})=0$ iff at least on of the conditions of Lemma 4 holds. Hence it is plausible to assume that the sign changes when the BMZ-collection moves over a position where $\mathcal{R}-z$ has an affine Tverberg point, or if one of the partition sets of $\mathcal{R}-z$ is affinely dependent, or, finally, if $\mathcal{R}-z$ has a Tverberg direction. However, the movement must be sufficiently generic, otherwise the collection could only "reflect" and the sign would not change. We did not attempt to describe such a generic movement precisely since we are not aware of convincing consequences $^{4}$ (except for the discussion in this section).

First we set $R_{1}:=\left\{c_{1}, c_{3}, c_{5}\right\}, R_{2}:=\left\{c_{2}, c_{4}, c_{6}\right\}$, and $R_{3}:=\{z\}$. In this case $\operatorname{sgn} \mathcal{R}=0$ iff at least one of the triangles $c_{1} c_{3} c_{5}$ or $c_{2} c_{4} c_{6}$ is degenerate. Thus a reasonable guess is that the sign depends only on the cyclic orientations of the triangles $c_{1} c_{3} c_{5}$ and $c_{2} c_{4} c_{6} .^{5}$

\footnotetext{
${ }^{4}$ If there were a direct definition of sign using this property then it would be surely of our interest.

${ }^{5}$ As we pointed out above, we do not have a precise proof. However, our observation is also supported by a computer program for computing the sign on many examples. A similar remark also applies for other choices of $\mathcal{R}$.
} 

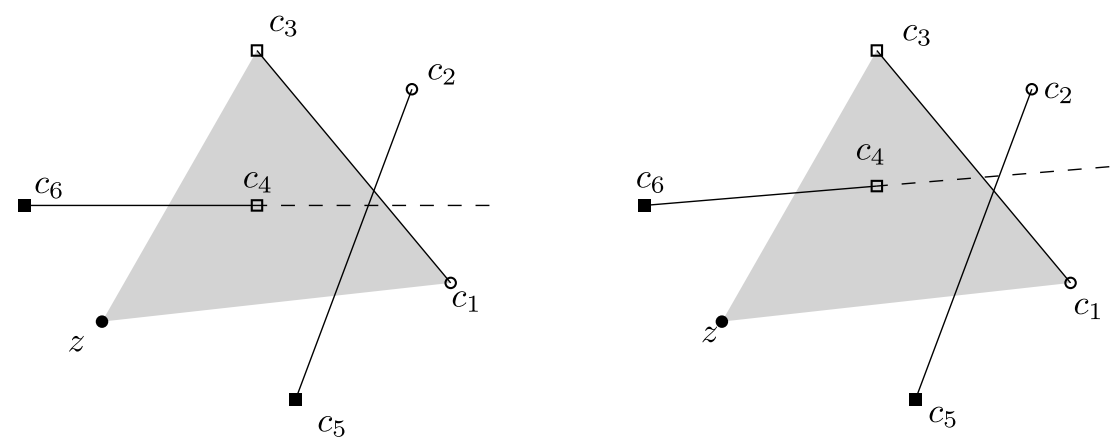

Fig. 9 The degree of this partition changes when the three lines pass through a common point

For $R_{1}:=\left\{c_{1}, c_{3}, c_{6}\right\}, R_{2}:=\left\{c_{2}, c_{4}\right\}, R_{3}:=\left\{c_{5}, z\right\}$, the situation is similar. The sign depends only on the cyclic orientation of the triangles $c_{1} c_{3} c_{6}$ and $c_{2} c_{4} c_{5}$.

Finally, let $R_{1}:=\left\{c_{2}, c_{5}\right\}, R_{2}:=\left\{c_{4}, c_{6}\right\}, R_{3}:=\left\{c_{1}, c_{3}, z\right\}$. Then the sign depends on the orientation of the lines $c_{2} c_{5}, c_{4} c_{6}$ and $c_{1} c_{3}$. However, it also depends on the mutual position of these lines, and it changes when all three of them pass through a common point. See Fig. 9.

Unfortunately, we are not aware of a simple uniform description of the three cases above.

Acknowledgements We would like to thank Marek Krčál for useful discussions at initial stages of this research. We also thank Günter M. Ziegler for valuable comments, and Peter Landweber and two anonymous referees for detailed comments and corrections that greatly helped to improve the presentation. In particular, we are indebted to one of the referees for pointing out to us reference [19].

M. Tancer is supported by the grants SVV-2010-261313 (Discrete Methods and Algorithms) and GAUK 49209.

U. Wagner's research is supported by the Swiss National Science Foundation (SNF Projects 200021125309 and 200020-125027).

\section{References}

1. Bajmóczy, E.G., Bárány, I.: A common generalization of Borsuk's and Radon's theorem. Acta Math. Hung. 34, 347-350 (1979)

2. Bárány, I., Larman, D.G.: A colored version of Tverberg's theorem. J. Lond. Math. Soc. 45(2), 314320 (1992)

3. Bárány, I., Shlosman, S.B., Szúcs, A.: On a topological generalization of a theorem of Tverberg. J. Lond. Math. Soc. 23, 158-164 (1981)

4. Bárány, I., Füredi, Z., Lovász, L.: On the number of halving planes. Combinatorica 10(2), 175-183 (1990)

5. Björner, A., Lovász, L., Vrećica, S.T., Živaljević, R.T.: Chessboard complexes and matching complexes. J. Lond. Math. Soc. 49(1), 25-39 (1994)

6. Blagojević, P.V.M., Matschke, B., Ziegler, G.M.: Optimal bounds for the colored Tverberg problem. Preprint (2009). http://arxiv.org/abs/0910.4987

7. Blagojević, P.V.M., Matschke, B., Ziegler, G.M.: Optimal bounds for a colorful Tverberg-Vrećica type problem. Adv. Math. 226, 5198-5215 (2011)

8. Grünbaum, B.: Convex Polytopes, 2nd edn. Graduate Texts in Mathematics, vol. 221. Springer, New York (2003) 
9. Kalai, G.: Combinatorics and convexity. In: Proceedings of the International Congress of Mathematicians, vols. 1, 2, pp. 1363-1374, Zürich, 1994. Birkhäuser, Basel (1995)

10. Matoušek, J.: Note on the colored Tverberg theorem. J. Comb. Theory, Ser. B 66(1), 146-151 (1996)

11. Matoušek, J.: Lectures on Discrete Geometry. Springer, New York (2002)

12. Matoušek, J.: A combinatorial proof of Kneser's conjecture. Combinatorica 24(1), 163-170 (2004)

13. Sarkaria, K.S.: A generalized van Kampen-Flores theorem. Proc. Am. Math. Soc. 111, 559-565 (1991)

14. Sarkaria, K.S.: Tverberg's theorem via number fields. Isr. J. Math. 79, 317-320 (1992)

15. Tverberg, H.: A generalization of Radon's theorem. J. Lond. Math. Soc. 41, 123-128 (1966)

16. Tverberg, H.: A generalization of Radon's theorem. II. Bull. Aust. Math. Soc. 24, 321-325 (1981)

17. Vrećica, S.T., Živaljević, R.T.: Chessboard complexes indomitable. Preprint (2009). http://arxiv.org/ abs/0911.3512

18. Ziegler, G.M.: Generalized Kneser coloring theorems with combinatorial proofs. Invent. Math. 147, 671-691 (2002); Erratum ibid. 163, 227-228 (2006)

19. Živaljević, R.T.: In pursuit of colored Carathéodory-Bárány theorems. Publ. Inst. Math. 57(71), 91100 (1995)

20. Živaljević, R.T.: User's guide to equivariant methods in combinatorics. II. Publ. Inst. Math. 64(78), 107-132 (1998)

21. Živaljević, R.T., Vrećica, S.T.: The colored Tverberg's problem and complexes of injective functions. J. Comb. Theory, Ser. A 61(2), 309-318 (1992) 